\title{
Überall trennscharfe Tests und monotone Dichtequotienten
}

\author{
Von
}

\section{J. Pfangagl*}

Gegeben sei ein Maßraum $(\mathfrak{X}, \mathscr{A}, \mu), \mu \sigma$-endlich, sowie eine Klasse $\mathfrak{B}$ von Wahrscheinlichkeitsmaßen, die von $\mu$ dominiert wird (d.h. alle $P \in \mathfrak{R}$ sind $\mu$-stetig). Dann existiert nach dem Theorem von RAdon-Nikodym zu jedem $P \in \mathfrak{P}$ eine Dichte bezüglich $\mu$, die wir mit $p$ bezeichnen wollen.

In [2] wurde gezeigt: Wenn es für eine Hypothese $P=P_{0}$ zu jeder Irrtumswahrscheinlichkeit $\alpha$ einen überall trennscharfen Test gibt (d. h. einen Test, der trennscharf gegen alle Alternativen $P \in \mathfrak{P}, P \neq P_{0}$ ist), dann kann der Dichtequotient $p(x) / p_{0}(x) P_{0}-$ f. ü. als monotone Funktion einer von der Alternative $P$ unabhängigen Funktion $T(x)$ dargestellt werden.

Die folgenden Ausführungen bringen eine Verallgemeinerung dieses Satzes für den Fall, daß der überall trennscharfe Test von der Hypothese unabhängig ist. (Die geläufigen Beispiele überall trennscharfer Tests haben alle diese Eigenschaft.) Es liegt nahe, zu vermuten, daß es in diesem Falle eine nicht nur von der Alternative, sondern auch von der Hypothese unabhängige Funktion $T(x)$ gibt, so daß die Dichtequotienten monotone Funktionen von $T(x)$ sind. Um diese Vermutung präzise formulieren zu können, müssen wir aber zunächst noch verschiedene Begriffe einführen.

Unter einer Testfunktion $\Phi(x)$ verstehen wir eine $\mathscr{A}$-meßbare Funktion, deren Wertevorrat in $[0,1]$ liegt. Die Interpretation von $\Phi$ als Wahrscheinlichkeit für die Verwerfung einer Hypothese legt folgende Definitionen nahe: $E_{P} \Phi(X)$ heißt Irrtumswahrscheinlichkeit von $\Phi$ bezüglich der Hypothese $P$. Die Testfunktion $\Phi_{0}$ heißt trennscharf für $P_{0}: P_{1}$ (d. h. trennscharf für die Hypothese $P_{0}$ gegen die Alternative $P_{1}$ ), wenn für alle Testfunktionen $\Phi$ mit $E_{0} \Phi \leqq E_{0} \Phi_{0}$ und $E_{1} \Phi \geqq$ $\geqq E_{1} \Phi_{0}$ gilt: $E_{0} \Phi=E_{0} \Phi_{0}, E_{1} \Phi=E_{1} \Phi_{0} \star \star$.

Diese Definition der Trennschärfe weicht von der üblichen insoferne ab, als eine Testfunktion $\Phi_{0}$ mit $E_{1} \Phi_{0}=1$ nur dann als trennscharf gilt, wenn der Wert 1 für $P_{1}$ nicht auch mit einer kleineren Irrtumswahrscheinlichkeit erreichbar ist, d. h. wenn es keine Testfunktion $\Phi$ gibt, für die $E_{1} \Phi=1$ und $E_{0} \Phi<E_{0} \Phi_{0}$. Dieser (strengere) Begriff der Trennschärfe liegt nicht nur intuitiv nahe; er beseitigt auch eine gewisse Asymmetrie zwischen $P_{0}$ und $P_{1}$ und erlaubt damit, verschiedene Formulierungen übersichtlicher zu gestalten, so z. B. beim Fundamentallemma von Neyman und Pearson [vgl. [1], S. 65, Theorem 1 (iii)] oder bei Hilfssatz 1 und 2. Die Symmetrie, die bei dieser Definition zwischen $P_{0}$ und $P_{1}$ besteht, zeigt sich auch darin, daß nun $\Phi_{0}$ genau dann trennscharf für $P_{0}: P_{1}$ ist, wenn $1-\Phi_{0}$ trennscharf für $P_{1}: P_{0}$ ist.

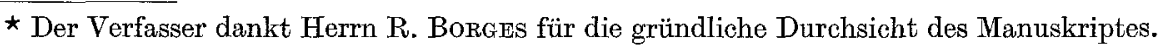
Seine kritischen Bemerkungen und die mit ihm geführten Diskussionen haben wesentlich zur Vereinfachung der Beweise beigetragen.

$\star \star$ Zur Vereinfachung schreiben wir $E_{p}$ statt $E_{P \nu}$.

Z. Wahrscheinlichkeitstheorie, Bd. 1 
Es sei jedoch nachdrücklich darauf hingewiesen, daß diese strengere Fassung des Begriffes der Trennschärfe keine entscheidende Rolle für die Gültigkeit der folgenden Sätze spielt. Die strengere Fassung wurde lediglich deshalb gewählt, weil sie, wie oben ausgeführt, gewisse Formulierungen übersichtlicher macht und außerdem intuitiv naheliegt.

Im folgenden setzen wir voraus, daß die Klasse $\mathfrak{F}$ total geordnet ist, derart, daß zwischen zwei verschiedenen Wahrscheinlichkeitsmaßen $P_{0}, P_{1} \in \mathfrak{B}$ genau eine der Relationen , $P_{0}<P_{1}$ " und , $P_{0}>P_{1}$ " besteht.

Wir nennen eine Testfunktion überall trennscharf in $\mathfrak{B}$, wenn sie trennscharf für alle $P_{0}: P_{1}$ mit $P_{0}<P_{1}$ ist, sobald $E_{0} \Phi>0$ und $E_{1} \Phi<1$; allenfalls könnte man noch zusätzlich verlangen, daß ein überall trennscharfer Test für mindestens ein Paar $P_{0}, P_{1} \in \mathfrak{F}$ trennscharf sein soll. Diese Modifikation spielt jedoch in den folgenden Überlegungen keine Rolle.

Eine Familie $\boldsymbol{\Phi}$ von Testfunktionen nennen wir perfekt bezüglich $\mathfrak{\Re}$, wenn alle Testfunktionen von $\boldsymbol{\Phi}$ überall trennscharf in $\mathfrak{F}$ sind und wenn es zu jedem $P_{0} \in \mathfrak{S}$ und jeder Irrtumswahrscheinlichkeit $\alpha \in[0,1]$ eine Testfunktion $\Phi \in \Phi$ gibt, so daß $E_{0} \Phi=\alpha$. Für $\alpha=\left\{\begin{array}{l}0 \\ 1\end{array}\right.$ müssen wir noch die Existenz eines $\Phi \in \boldsymbol{\Phi}$ explizite fordern, das trennscharf ist für alle $\left\{\begin{array}{l}P_{0}: P \\ P: P_{0}\end{array}\right.$ mit $\begin{array}{l}P>P_{0} \\ P<P_{0}\end{array}$, da diese Fälle in der Definition von überall trennscharf ausdrücklich ausgenommen wurden.

Wir sagen, die Klasse $\mathfrak{B}$ besitzt monotone Dichtequotienten, wenn eine $\mathscr{A}$ meßbare reellwertige Funktion $T(x)$ existiert, derart, daß für alle $P_{0}, P_{1} \in \mathfrak{F}$, $P_{0}<P_{1}$, der Dichtequotient $p_{1}(x) / p_{0}(x)$ eine monoton steigende Funktion von $T$ ist, genauer: Wenn es eine monoton nicht fallende Funktion $H$ gibt, so daß

$$
p_{1}(x) / p_{0}(x)=H(T(x)) \quad\left(P_{1}+P_{0}-\text { f. ̈̈. }\right)
$$

Dabei wollen wir für $H$ auch den Funktionswert $\infty$ zulassen. (Die Funktion $H$ hängt - im Gegensatz zu $T$ - natürlich von den speziellen Wahrscheinlichkeitsmaßen $P_{0}, P_{1}$ ab.)

Satz: Zu einer total geordneten und dominierten Klasse $\mathfrak{F}$ von Wahrscheinlichkeitsmaßen gibt es dann und nur dann eine perfekte Familie von Testfunktionen, wenn $\$$ monotone Dichtequotienten besitzt.

Bevor wir den Beweis führen, seien einige Bemerkungen vorweggeschickt.

Daß die Monotonie der Dichtequotienten hinreichend für die Existenz einer perfekten Familie von Testfunktionen ist, liegt auf der Hand und findet sich im wesentlichen bereits bei LEHMAnN ([1], S. 68, Theorem 2). Da aber die Formulierung bei LEHMANN nicht ganz mit der unserigen identisch ist und außerdem der Beweis bei LEHIANN auf den Fall streng monotoner $H$ abgestellt ist, wird weiter unten auch der Beweis für diesen Teil der Behauptung skizziert.

Die Betonung des Satzes liegt jedoch darauf, daß die Monotonie der Dichtequotienten auch notwendig für die Existenz einer perfekten Familie von Testfunktionen ist.

$\mathrm{Da}$ \$ nach Voraussetzung dominiert ist, gibt es nach einem bekannten Lemma von Halmos und L. J. Savage (vgl. z. B. [1] S. 354, Theorem 2) auch ein Maß

$$
Q=\sum a_{i} Q_{i}, \quad \sum a_{i}=1, \quad Q_{i} \in \mathfrak{F},
$$


das die Klasse $\$$ dominiert. Aus (2) folgt aber sofort, daß die Dichten bezüglich $Q$ alle die Gestalt $\left(\sum a_{i} q_{i}(x) / p(x)\right)^{-1}$ haben, im Falle monotoner Dichtequotienten also $Q-\mathrm{f}$. ủ. Funktionen von $T$ sind. Wir können dahor jede geordnete und dominierte Klasse von Wahrscheinlichkeitsmaßen, für die es eine perfekte Familie von Testfunktionen gibt, durch geeignete Wahl des MaBraumes zurückführen auf eine geordnete Klasse von Wahrscheinlichkeitsmaßnahmen über $\operatorname{dem} R_{1}$, wobei der Dichtequotient $p_{1}(t) / p_{0}(t)$ für $P_{0}<P_{1}$ eine monoton nicht fallende Funktion von $t$ ist. Dies zeigt, daB geordnete und dominierte Klassen von Wahrscheinlichkeitsmaßen, für die es eine perfekte Familie von Testfunktionen gibt, eine sehr spezielle Struktur haben.

Um die statistische Bedeutung obigen Satzes zu diskutieren, führen wir in Anlehnung an Theorem 2 (iv) bei Lenmann ([1] S. 68/9) den Begriff der optimalen Testfunktion ein.

Eine Testfunktion $\Phi_{0}$ heißt für $P_{0}$ optimal (in $\mathfrak{B}$ ), wenn für alle Testfunktionen $\Phi$ mit $E_{0} \Phi=E_{0} \Phi_{0}$ gilt:

$$
E \Phi \leqq E \Phi_{0} \text {, für alle } P \in \Re \text { mit } P \gtrless P_{0} .
$$

Eine für $P_{0}$ optimale Testfunktion maximisiert nicht nur die Ablehnwahrscheinlichkeit für alle $P>P_{0}$; sie maximisiert gleichzeitig auch die Annahmewahrscheinlichkeit für alle $P<P_{0}$, beides unter Einhaltung einer vorgegebenen Irrtumswahrscheinlichkeit für $P_{0}$. Wie leicht einzusehen ist, folgt daraus, daß eine für $P_{0}$ optimale Testfunktion $\Phi_{0}$ trennscharf für alle $P_{1}: P_{2}$ mit $P_{1} \leqq P_{0}$ und $P_{2} \geqq P_{0}$ ist, soferne $E_{1} \Phi_{0}>0$ und $E_{2} \Phi_{0}<1$. Sie liefert daher im gewissen Sinne eine optimale Lösung der Aufgabe, die (zusammengesetzte) Hypothese $P \leqq P_{0}$ gegen die (zusammengesetzte) Alternative $P>P_{0}$ zu testen.

Offenbar ist eine in $\mathfrak{F}$ überall trennscharfe Testfunktion $\Phi_{0}$ für alle jene $P_{0} \in \mathfrak{F}$ optimal (in $\mathfrak{B}$ ), für die $E_{0} \Phi_{0} \in(0,1)$ und umgekehrt: Ist eine Testfunktion $\Phi_{0}$ optimal (in $\mathfrak{B}$ ) für alle $P_{0} \in \mathfrak{S}$ mit $E_{0} \Phi_{0} \in(0,1)$, so ist sie in $\mathfrak{P}$ überall trennscharf.

Bisher haben wir die Beziehung zwischen optimalen und überall trennscharfen Testfunktionen untersucht. Nun untersuchen wir die Beziehung zwischen Frmilien von optimalen und Familien von überall trennscharfen Testfunktionen. Es ist naheliegend, in Analogie zur perfekten Familie von Testfunktionen eine Familie von optimalen Testfunktionen zu betrachten, die zu jedem $P \in \mathfrak{P}$ und zu jeder Irrtumswahrscheinlichkeit $\alpha \in[0,1]$ eine für $P$ optimale Testfunktion $\Phi$ mit der Irrtumswahrscheinlichkeit $E_{P} \Phi=\alpha$ enthält. Offenbar hat eine perfekte Familie von Testfunktionen diese Eigenschaft, denn es gibt zu jedem $P_{0} \in \mathbb{B}$ und zu jedem $\alpha \in(0,1)$ eine Testfunktion $\Phi_{0}$ mit $E_{0} \Phi_{0}=\alpha$, welche überall trennscharf und daher entsprechend den obigen Ausführungen auch optimal für alle $P \in \mathfrak{B}$ mit $E \Phi_{0} \in(0,1)$, insbesondere also auch optimal für $P_{0}$ ist. Für $\alpha=0$ und $\alpha=1$ gibt es nach Definition des Begriffes „perfekt" gleichfalls optimale Testfunktionen.

Man kann aber auch zeigen, daß umgekehrt jede Familie von Testfunktionen, die zu jedem $P_{0} \in \mathfrak{S}$ und zu jedem $\alpha \in[0,1]$ eine optimale Testfunktion $\Phi_{0}$ mit $E_{0} \Phi_{0}=\alpha$ enthält, perfekt ist. Wie bereits oben betont wurde, ist klar, daß $\Phi_{0}$ trennscharf für alle $P_{1}: P_{2}$ mit $P_{1} \leqq P_{0}$ und $P_{2} \geqq P_{0}$ ist, so ferne $E_{1} \Phi_{0}>0$ und $E_{2} \Phi_{0}<1$. Wir haben noch zu zeigen, daß $\Phi_{0}$ auch trennscharf für alle $P_{1}: P_{2}$ mit $P_{1}<P_{2}<P_{0}$ oder $P_{0}<P_{1}<P_{2}$ ist. Ohne Beschränkung der Allgemeinheit können wir $P_{0}<P_{1}<P_{2}$ annehmen. Nach Voraussetzung existiert für $P_{1}$ eine optimale Testfunktion $\Phi_{1}$ mit der Irrtumswahrseheinlichkeit $E_{1} \Phi_{0}>0$. (Dabei können wir den Fall $E_{1} \Phi_{0}=1$ als trivial von vorneherein außer acht lassen.) Da sowohl $\Phi_{0}$ als auch $\Phi_{1}$ optimal sind, folgt aus $E_{1} \Phi_{1}=E_{1} \Phi_{0}$, daß $E_{0} \Phi_{1}=E_{0} \Phi_{0}$. Nun ist aber wegen $P_{2}>P_{0}$ die Testfunktion $\Phi_{0}$ unter der Voraussetzung $E_{2} \Phi_{0}<1$ trennscharf für $P_{0}: P_{2}$ und wegen $P_{0}<P_{1}<P_{2}$ auch $\Phi_{1}$ tremscharf für $P_{0}: P_{2}$, so daß $E_{2} \Phi_{1}=E_{2} \Phi_{0}$. Da $\Phi_{1}$ trennscharf für $P_{1}: P_{2}$ ist, folgt daraus aber, daß auch $\Phi_{0}$ trennscharf für $P_{1}: P_{2}$ ist, q.e.d. 
Zusammen mit dem oben behaupteten Satz folgt aus den bisherigen Ausführungen also, daß die Aufgabe, die (zusammengesetzte) Hypothese $P \leqq P_{0}$ gegen die (zusammengesetzte) Alternative $P>P_{0}$ zu testen, bei dominiertem $\mathfrak{P}$ dann und nur dann eine optimale Lösung hat, wenn $\mathfrak{P}$ monotone Dichtequotienten besitzt. Die Voraussetzung, daß die Lösung o ptimal sein soll, ist dabei wesentlich. Verlangen wir nur, daß es zu jedem $P_{0} \in \mathfrak{S}$ und jeder Irrtumswahrscheinlichkeit $\alpha \in[0,1]$ eine Testfunktion $\Phi_{0}$ geben soll, die trennscharf für alle $P_{0}: P$ mit $P>P_{0}$ ist und für die $E \Phi_{0} \leqq \alpha$ für alle $P \leqq P_{0}$ (ohne zu verlangen, daß für $P<P_{0}$ gilt $E \Phi_{0} \leqq E \Phi$ für alle $\Phi$ mit $E_{0} \Phi=\alpha$ ), so ist die Voraussetzung monotoner Dichtequotienten keineswegs notwendig für die Existenz einer Lösung. Dies zeigt folgendes Beispiel:

Es sei $c \in(0,1)$ eine beliebige Konstante. Wir definieren die Familie $\mathfrak{P}$ durch ihre Dichten bezüglich des Lebesgueschen Maßes in $[0,1]$ :

$$
p_{\vartheta}(x)=\left\{\begin{array}{cl}
c+(1-c) / \vartheta & \text { für } 0 \leqq x \leqq \vartheta \\
c & \text { für } \vartheta<x \leqq 1
\end{array}\right.
$$

Wir definieren nun die Testfunktion $\Phi_{\vartheta}(x ; \alpha)$ :

$$
\begin{array}{ll}
0 \leqq \alpha \leqq c(1-\vartheta) & \Phi_{\vartheta}(x ; \alpha)= \begin{cases}1 \text { für } \vartheta \leqq x \leqq \vartheta+\alpha / c \\
0 \text { sonst }\end{cases} \\
c(1-\vartheta)<\alpha \leqq 1 & \Phi_{\vartheta}(x ; \alpha)= \begin{cases}1 \text { für } \frac{1-\alpha}{\mathrm{c}+(1-\mathrm{c}) / \vartheta} \leqq x \leqq 1 \\
0 \text { sonst }\end{cases}
\end{array}
$$

Die Testfunktion $\Phi_{\vartheta_{0}}$ ist trennscharf für alle $p_{\vartheta_{0}}: p_{\vartheta^{\prime}}$ mit $\vartheta>\vartheta_{0}$; außerdem gilt: $E_{\vartheta} \Phi_{\vartheta_{0}}(X ; \alpha) \leqq \alpha$ für alle $\vartheta \leqq \vartheta_{0}$. Es gibt also für die oben angegebene Klasse von Verteilungsfunktionen zu jedem $\vartheta_{0}$ und zu jeder Irrtumswahrscheinlichkeit einen Test für die Hypothese $\vartheta \leqq \vartheta_{0}$, der trennscharf gegen alle $\vartheta>\vartheta_{0}$ ist. Diese Klasse von Verteilungsfunktionen besitzt aber, wie man leicht nachprüft, keine monotonen Dichtequotienten.

Nun beweisen wir, daß die Monotonie der Dichtequotienten hinreichend für die Existenz einer perfekten Familie von Testfunktionen ist.

Zu diesem Zwecke bilden wir die Funktion $\lambda(c)=Q(T(X)>c)$ für $-\infty \leqq c \leqq \infty$. Die Funktion $\lambda(c)$ ist monoton nicht wachsend und rechtsseitig stetig. Es gibt also zu jedem Wert $\lambda \in[0,1]$ ein $c_{0} \in[-\infty,+\infty]$, so $\operatorname{da} \beta \lambda\left(c_{0}\right) \leqq \lambda \leqq \lambda\left(c_{0}-0\right)$. Wir definieren:

$$
\Phi_{\lambda}(x)=\left\{\begin{array}{l}
0 \\
\lambda-\lambda\left(\mathrm{c}_{0}\right) \\
\frac{\lambda\left(\mathrm{c}_{0}-0\right)-\lambda\left(\mathrm{c}_{0}\right)}{\text { für }} \mathrm{T}(x) \stackrel{<}{=} c_{0} \\
1
\end{array}\right\}^{\star}
$$

Nun zeigen wir, daß $\boldsymbol{\Phi}=\left\{\Phi_{\lambda}: \lambda \in[0,1]\right\}$ eine perfekte Familie von Test. funktionen ist. Es gilt:

$\int \Phi_{\lambda}(x) d Q=\lambda$ für alle $\lambda \in[0,1] . \quad$ Da $\Phi_{\lambda}(x) \rightarrow \Phi_{\lambda_{0}}(x) \quad(Q-$ f.ü. $)$ für $\lambda \rightarrow \lambda_{0}$ und da $P \ll Q$ für alle $P \in \mathfrak{P}$, ist $E_{P} \Phi_{\lambda}(x)$ eine stetige Funktion von $\lambda$, die die

* Falls $\lambda\left(c_{0}-0\right)=\lambda\left(c_{0}\right)$ ist die Definition von $\Phi_{2}(x)$ für $T(x)=c_{0}$ ohne Belang. 
Werte 0 und 1 und daher auch alle Zwischenwerte annimmt. Es gibt also zu jedem $P \in \mathfrak{B}$ and zu jeder Trrtumswahrscheinlichkeit $\alpha \in[0,1]$ eine Testfunktion $E \Phi_{\lambda}=\alpha$. Diese Testfunktionen $\Phi_{\lambda}$ sind überall trennscharf in $\Re$ : Es seien $P_{0}, P_{1} \in \mathfrak{B}$ mit $P_{0}<P_{1}$ gegeben, so daß

$$
E_{0} \Phi_{\lambda}>0 \text { und } E_{1} \Phi_{\lambda}<1
$$

Weiter gilt nach Voraussetzung die Relation (1). Wegen der Monotonie von $H$ folgt aus $H(T(x)) \lesseqgtr H\left(c_{0}\right)$, daB $T(x) \lesseqgtr c_{0}$. Daher ergibt sich aus $(3)$ :

$$
\Phi_{\lambda}(x)=\left\{\begin{array}{l}
0 \\
1
\end{array} \text { für } H(T(x)) \lesseqgtr H\left(c_{0}\right)\right.
$$

Daraus folgt aber nach dem Fundamentallemma von Neyman und Pearsox (vgl. z. B. [1] S. 65, Theorem 1 (ii)) wegen (4) sofort, daß $\Phi_{\lambda}$ trennscharf für $P_{0}: P_{1}$ ist. Es sei $\lambda_{0}=\inf \left\{\lambda: E_{0} \Phi_{\lambda}>0\right\}$. Da es zu jedem $\alpha \in(0,1)$ ein $\Phi_{\lambda}$ mit $E_{0} \Phi_{\lambda}=\alpha$ gibt, folgt aus der Trennschärfe dieser $\Phi_{\lambda}$ und der Stetigkeit von $\Phi_{\lambda}$ in $\lambda$, daß $E_{0} \Phi_{\lambda_{0}}=0$ ist and $\Phi_{\lambda_{0}}$ trennscharf für $P_{0}: P_{1}$ für alle $P_{1}>P_{0}$ ist. Ebenso zeigt man die Existenz eines $\lambda_{1}$ mit $E_{1} \Phi_{\lambda_{1}}=1$, so daß $\Phi_{\lambda_{1}}$ trennscharf für $P_{0}: P_{1}$ für alle $P_{0}<P_{1}$ ist.

Nun beweisen wir, daß die Existenz einer perfekten Familie von Testfunktionen hinreichend für die Monotonie der Dichtequotienten ist.

Jedem Wahrscheinlichkeitsmaß $P \in \mathfrak{P}$ ordnen wir folgende Mengen zu:

$$
\begin{aligned}
& P^{*}=\{x: p(x)>0\} \\
& P^{-}=\overline{P^{*}} \bigcup_{Q_{j}<P} Q_{j}^{*} \\
& P^{+}=\overline{P^{*}} \bigcup_{Q_{j}>P} Q_{i}^{*}
\end{aligned}
$$

Wir bemerken, daB bei Existenz einer perfekten Familie von Testfunktionen $P_{0}^{-} \subset P_{1}^{-}$und $P_{0}^{+} \supset P_{1}^{+}$für $P_{0}<P_{1}$ gilt. Wir verzichten auf einen Beweis, da wir diese Relation nicht benötigen.

Jeder Testfunktion $\Phi$ ordnen wir den Annahmebereich $C_{\Phi}$ und den Ablehnbereich $C_{\emptyset}^{\prime} \mathrm{zu}$ :

$$
C_{\phi}=\{x: \Phi(x)=0\}, \quad C_{\Phi}^{\prime}=\{x: \Phi(x)=1\}
$$

Wir nennen einen Annahmebereich $C$ trennscharf für $P_{0}: P_{1}$, wenn es in $\Phi$ eine für $P_{0}: P_{1}$ trennscharfe Testfunktion mit dem Annahmebereich $C$ gibt.

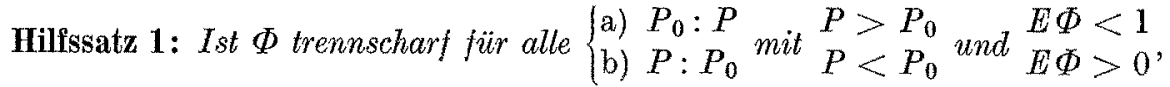
dann gill $Q-$ f. ü.:
a) $P_{0}^{+} \subset C_{\Phi}^{\prime}$
b) $P_{0}^{-} \subset C_{\Phi}$.

Beweis: Unter Voraussetzung a) folgt aus der Trennschärfe von $\Phi$ für alle $Q_{j}>P_{0}$ mit $E_{Q_{j}} \Phi<1$ :

$$
\bar{P}_{0}^{*} Q_{j}^{*} \subset C_{\Phi}^{\prime} \quad(Q-\mathbf{f} . \ddot{\mathrm{u}} .)
$$

Für $E_{Q_{j}} \Phi=1$ gilt diese Relation in trivialer Weise. Indem wir die Vereinigung über alle $Q_{j}>P_{0}$ bilden, erhalten wir daraus a). Der Beweis für b) verläuft analog, wobei hier die schärfere Definition der Trennschärfe wesentlich ist. 
Korollar I: Gelten sowohl Voraussetzung a) als auch b), so ist

$$
C_{\Phi}=P_{0}^{-} \cup C_{\Phi} P_{0}^{*} \quad(Q-\mathbf{f} . \text { ü. })
$$

Korollar I: Ist $\Phi$ überall trennscharf in $\mathfrak{P}$, so gilt $Q-$ f. ü. für beliebige $P_{0} \in \mathfrak{\Re}$ :
a) $C_{\Phi} \subset P_{0}^{-}$
wenn $P_{0}\left(C_{\Phi}\right)=0$
b) $C_{\Phi} \supset P_{0} \cup P_{0}^{*}$ wenn $P_{0}\left(C_{\Phi}\right)=1$.

Das letzte Korollar folgt aus Hilfssatz 1, indem man die Gültigkeit von Voraussetzung a) bzw. b) zeigt.

Es sei $\mathscr{C}$ die Klasse aller Bereiche

$$
A=P_{0}^{-} \cup\left\{x: p_{1}(x) \leqq c p_{0}(x)\right\} P_{0}^{*}
$$

für beliebige $P_{0}, P_{1} \in \mathfrak{F}$ mit $P_{0}<P_{1}$ und beliebiges $c \in[0, \infty]$.

Jede Menge $A \in \mathscr{C}$ ist $Q$ - f.ü. mit dem Annahmebereich einer überall trennscharfen Testfunktion $\Phi \in \boldsymbol{\Phi}$ identisch. Nach Definition von $\boldsymbol{\Phi}$ gibt es nämlich sicher eine Testfunktion $\Phi \in \Phi$ mit der Irrtumswahrscheinlichkeit $1-P_{0}(A)$, die sowohl Voraussetzung a) als auch b) von Hilfssatz 1 erfüllt. (Im Falle $P_{0}(A)$ $=\left\{\begin{array}{l}0 \\ 1\end{array}\right.$ wird die Existenz eines $\Phi$, das Voraussetzung $\left\{\begin{array}{l}a \\ b\end{array}\right)$ erfüllt, in der Definition von $\boldsymbol{\Phi}$ explizit gefordert.) Also gilt nach Korollar I:

$$
C_{\Phi}=P_{0}^{-} \cup C_{\Phi} P_{0}^{*} \quad(Q-\mathrm{f} . \ddot{\mathrm{u}} .)
$$

Da $\Phi$ trennscharf (im schwächeren, d. h. üblichen Sinne) für $P_{0}: P_{1}$ mit der Irrtumswahrscheinlichkeit $E_{0} \Phi=1-P_{0}(A)$ ist, folgt wegen

$$
A=\left\{x: p_{1}(x) \leqq c p_{0}(x)\right\} \quad\left(P_{0}-\text { f.ü. }\right),
$$

nach dem Fundamentallemma von Neymar und Pearson, daß $A=C_{\Phi} P_{0}-\mathrm{f}$. ü. Wegen (7) und (8) gilt diese Gleichheit auch $Q-\mathrm{f}$. ü.

Hilissatz 2: Die Klasse $\mathscr{C}$ ist $Q-\mathrm{f}$.ü. geordnet, d. h. für zwei beliebige Mengen $A, B \in \mathscr{C}$ gill:

$$
A \subset B(Q-\text { f.ü. }) \text { oder } B \supset A(Q-\text { f.ü. }) \text {. }
$$

Beweis: Der Bereich $A$ sei in der Form (7) gegeben. Wie wir uns oben überlegt haben, ist $B$ der Annahmebereich einer überall trennscharfen Testfunktion. Ist $P_{0}(B)=0$ oder 1 , so ist die Ordnungsrelation zwischen $A$ und $B$ im Hinblick auf Korollar II evident. Für $P_{0}(B) \in(0,1)$ ist $B$ trennscharf (im schwächeren Sinne) für $P_{0}: P_{1}$. Es gibt also wegen (9) nach dem Fundamentallemma von Nwyman und Pearson ein $c_{B}$, so daß

$$
\left\{x: p_{1}(x)<c_{B} p_{0}(x)\right\} \subset B \subset\left\{x: p_{1}(x) \leqq c_{B} p_{0}(x)\right\} \quad\left(P_{0}-\text { f. ̈̈. }\right) .
$$

Daher sind $A$ und $B P_{0}-$ f.ü. geordnet. Außerdem gilt in diesem Falle sowohl Voraussetzung a) als auch b), so daß $B$ nach Korollar I $Q-$ f.ü. in der Form $B=P_{0}^{-} \cup B P_{0}^{*}$ dargestellt werden kann. Wegen (7) folgt daraus aber, daß die Ordnung, die bisher nur $P_{0}-$ f.ü. nachgewiesen war, auch $Q-$ f. ü. gilt.

Da $\mathscr{C} Q-$ f.ü. geordnet ist, gibt es nach ([2] S. 171, Hilfssatz 1) eine $Q-$ f.ü. definierte reellwertige $A$-meßbare Funktion $T(x)$, so daß für alle $A \in \mathscr{C}$ gilt:

$$
A=\{x: T(x) \leqq Q(A)\} \quad(Q-\text { f. ü. })
$$


Wir betrachten nun für festes $P_{0}, P_{1} \in \$_{\$}, P_{0}<P_{1}$, die Schar:

$$
A_{0}=P_{0}^{-} \cup\left\{x: p_{1}(x) \leqq c p_{0}(x)\right\} P_{0}^{*}
$$

Da $Q \sigma$-additiv ist, folgt aus $A_{c_{0}}=\bigcap_{c>c_{0}} A_{c}$ sofort, da $Q\left(A_{c}\right)$ eine rechtsseitig stetige Funktion von $c$ ist. Außerdem ist $Q\left(A_{c}\right)$ offenbar monoton nicht fallend. Also ist

$$
H(y)= \begin{cases}\inf \left\{c: Q\left(A_{c}\right) \geqq y\right\} & \text { für } y \leq Q\left(A_{\infty}\right) \\ +\infty & \text { sonst }\end{cases}
$$

eine monoton nicht fallende und linksseitig stetige Funktion, derart, daß für $c<+\infty$

$$
y \leqq Q\left(A_{c}\right) \text { dann und nur dann, wenn } H(y) \leqq c .
$$

Daher folgt aus (10) für $c \in[0, \infty)$ :

$$
A_{c}=\{x: H(T(x)) \leqq c\} \quad Q-\mathrm{f} . \ddot{\mathrm{u}} .
$$

Durch Kombination mit (11) erhalten wir wegen $P_{0}^{*}=\left\{x: p_{0}(x)>0\right\}$ für alle $c \in[0, \infty)$ :

$$
P_{0}^{-} \cup\left\{x: p_{1}(x) / p_{0}(x) \leqq c\right\} P_{0}^{*}=\{x: H(T(x)) \leqq c\} \quad(Q-\text { f.ü. }) .
$$

Setzen wir

so erhalten wir:

$$
p_{1}(x) / p_{0}(x)=\left\{\begin{array}{rll}
0 & \text { für } & x \in P_{0}^{-} \\
\infty & \text { für } & x \in P_{0}^{+}
\end{array}\right.
$$

$$
p_{1}(x) / p_{0}(x)=H(T(x)) \quad(Q-\text { f. ü. })
$$

\section{Literatur}

[1] Lemmand, E. L.: Testing statistical hypotheses. New York: Wiley 1959.

[2] Pfanzact, J.: Über die Existenz überall trennscharfer Tests. Metrika 3, 169-176 (1960) und 4, 105-106 (1961).

Seminar für Wirtschafts- und Sozialstatistik, Universitat Köln,

5 Koln-Lindenthal, Albertus-Magnus-Platz 1 